\title{
Herramienta para la simulación del movimiento de un micro robot para aplicaciones médicas a partir de un arreglo de bobinas basadas en Maxwell - Helmholtz. (Herramienta de simulación para navegación de microrobots)
}

\author{
$\varnothing$ Martín Alonso Muñoz Medina $^{1}$ \\ OSCAR A. VIVAS ${ }^{1}$ \\ LEONARDO RICCOTTI ${ }^{2}$
}

\section{Resumen}

Objetivo: Los micro robots pueden ser movidos de varias maneras, una de ellas hace uso de señales magnéticas. Este artículo muestra un sistema de navegación magnético para mover y orientar un micro robot para un eventual uso médico, así como una herramienta gráfica para simular el movimiento de dicho micro robot.

Materiales y métodos: El artículo presenta inicialmente el proceso de fabricación de un micro robot a partir de diferentes concentraciones de polvo magnético sobre un polímero, su movimiento experimental utilizando un arreglo de bobinas, y la implementación de una herramienta gráfica que simula dicho movimiento.

Resultados: El micro robot fabricado pudo moverse en una pequeña arena experimental, variando su posición y orientación dependiendo de la corriente inyectada a dos bobinas que utilizan el arreglo de Maxwell - Helmholtz. La herramienta gráfica, que fue implementada en Unity 3D, mostró un comportamiento muy similar al real.

Conclusiones: La herramienta gráfica probó que puede simular con precisión el movimiento real de un micro robot movido a través de dos bobinas magnéticas que utilizan el arreglo de Maxwell - Helmholtz.

Palabras clave: Micro robots, Campos Magnéticos, Maxwell - Helmholtz, Simulación Gráfica.

1 Universidad del Cauca. Popayán, Colombia.

2 BioRobotics Institute, Scuola Superiore Sant'Anna, Pisa, Italia.

Autor de correspondencia: Muñoz Medina, M.A. (Martín Alonso): Calle 27BN 7-26 B/Palacé, Popayán, Colombia. Teléfono: (+57) 8209900 Ext. 2122/ 3113332127

Correo electrónico: maamunoz@unicauca.edu.co
Historia del artículo:

Artículo recibido: 8-II-2018/ Aprobado: 6-V-2018

Disponible online: 3 de agosto 2018

Discusión abierta hasta abril de 2020 


\section{Simulation tool for microrobot navigation in medical applications, based on Maxwell - Helmholtz coil array. (Simulation tool for microrobot navigation)}

\section{Abstract}

Objective: Microrobots can be operated in many ways, one of them is through magnetic fields. This paper shows a magnetic navigation system to move and direction a microrobot for future medical use, as well as a graphic tool for the simulation.

Material and method: This article presents at first the fabritation process of the microrobot given by different powder concentrations in a polymer, the experimentation movement by a coil array and the development of a graphic tool to simulate the behavior.

Results: The built microrobot could move in a small arena, changing its position and orientation depending of the amount of current applied to the coil array Maxwell - Helmholtz. The graphic tool, developed in Unity 3D, shows a behavior similar to the real one.

Conclusions: The graphic tool proved itself to simulate with high accuracy the movement of the real microrobot through the coil array Maxwell - Helmholtz.

Keywords: micro robots, magnetic field, Maxwell - Helmholtz, graphic simulation.

\section{Ferramenta para simulação do movimento de um micro robô para aplicações médicas, a partir de bobinas magnéticas baseadas no arranjo de Maxwell-Helmholtz}

\section{Resumo}

Objetivo: Micros robôs podem-se mover fazendo uso de sinais magnéticos. Este artigo amostra um sistema de navegação magnética para mover e orientar um micro robô de uso médico, além apresenta uma ferramenta gráfica para simular o movimento do micro robô.

Materiais e métodos: Neste artigo apresenta-se o processo de fabricação de um micro robô a partir de diferentes concentrações de pó magnético sobre um polímero. 0 movimento experimental do micro robô descreve-se utilizando um arranjo de bobinas e a simulação do seu movimento é apresentada através de uma ferramenta gráfica.

Resultados: 0 micro robô fabricado foi capaz de mover-se em uma pequena arena experimental, suas variações de posição e orientação dependeram da corrente injetada em duas bobinas construídas segundo o arranjo Maxwell - Helmholtz. A ferramenta gráfica foi implementada no Unity 3D e mostrou um comportamento muito semelhante ao real.

Conclusões: A ferramenta gráfica demonstrou sua capacidade para simular com precisão o movimento real de um micro robô, que é movido através de duas bobinas magnéticas que utilizam o arranjo Maxwell - Helmholtz.

Palavras-chave: micro robôs, campos magnéticos, Maxwell - Helmholtz, simulação gráfica. 


\section{Introducción}

La inclusión de robots dentro de la medicina quirúrgica abre un campo de convergencia entre diversas áreas, lo cual se traduce en un beneficio adicional para los pacientes (V Joseph et al., 2005). Con la aparición de los micro robots (máquinas basadas en la primera generación de robots pero con una considerable disminución en su tamaño), las aplicaciones crecen potencialmente, porque sin tener modificaciones importantes en su estructura funcional, conservan la movilidad y anatomía del modelo inicial. Una de estas, es la movilización de autómatas a través de cavidades corporales con el objeto de ejecutar procedimientos que antes parecían imposibles sin una laceración (Dario et al., 2000; Mack, 2001). En el futuro se espera que la evolución de estos dispositivos permita ejecutar funciones más complejas en múltiples intervenciones quirúrgicas.

La incidencia de una cápsula endoscópica en el diagnóstico gastrointestinal es un claro ejemplo de robótica aplicada. El reemplazo de la manguera por una píldora que contiene una cámara diminuta, ha disminuido considerablemente el trauma que sufren los pacientes con este incómodo procedimiento. Avances futuros apuntan a que la píldora incluya un mecanismo de recolección de tejido a partir de muestras, procedimiento conocido como biopsia, que sustituya completamente el método convencional (Menciassi et al., 2007).

A pesar de estar en una etapa de desarrollo muy temprana, con los micro robots ya se visualizan varios procedimientos médicos, entre ellos la administración objetiva de fármacos (Woods \& Constandinou, 2013), concentrando la cantidad de medicamento necesario sobre cierta área del cuerpo, optimizando el uso de la substancia y evitando efectos secundarios no deseados (Lai et al., 2014). En tratamientos de braquiterapia, será posible irradiar células cancerígenas desde adentro, posicionando un emisor cerca de las células no deseadas, como un tumor (Phillip \& Devlin, 2015). El beneficio frente a otras técnicas como la quimioterapia, es que el po- sicionamiento sería relativamente sencillo y no varía con el movimiento del paciente, disminuyendo considerablemente la exposición sobre otras áreas no deseadas. Actualmente los métodos más destacables para llevar a cabo dichos procesos usan campos magnéticos de alta frecuencia (Andrä \& Nowak, 2007), y resonancia ultrasónica (D.-H. Kim et al., 2004).

La remoción de material también se muestra con un panorama alentador, limpiando caminos, realizando cortes o en la obtención de muestras de tejido (Qiu et al., 2012). Debido a que el micro robot no es lo suficientemente grande para llevar herramientas, él en sí es la herramienta. La ablación (eliminación de un órgano o tejido mediante una intervención quirúrgica), es posible con motores rotatorios similares al funcionamiento de un taladro, por ejemplo en la remoción de grasas acumuladas en las vías circulatorias (Qiu et al., 2012). 0 también el uso de ondas de ultrasonido haría resonar mecánicamente el micro robot, destruyendo objetos determinados como un cálculo (Watson et al., 2009). Con el diseño adecuado, las biopsias (muestras de tejido) se extraerían del paciente para un posterior análisis (Menciassi et al., 2007). Futuras y más complejas tareas apuntan a que esos análisis puedan realizarse directamente en el ambiente donde se toma la muestra, apoyando la tarea con sensores y comunicación inalámbrica (Shah et al., 2009).

Utilizados como estructuras móviles, existe la capacidad de controlar el movimiento y ubicación de un puente entre células, tejidos o arterias, mediante el uso de viaductos microscópicos guiados por un micro robot (Luechinger et al., 2010; Zhang et al., 2005). El micro robot podría moverse a lo largo de un canal hasta posicionar una endoprótesis vascular, que son dispositivos tubulares que se colocan temporalmente sobre algún ducto, ayudando a sanar el tejido o manteniendo el canal libre de obstáculos (Saito et al., 2003); o bien realizando la oclusión intencional en una vía, de forma temporal o permanente, como una vena que alimenta un tumor (Ergeneman et al., 2008; Park et al., 2010). Finalmente un micro robot que pueda ubicarse de 
forma temporal o permanente en el cerebro para su estimulación (Chatzipirpiridis et al., 2015), podría capturar una señal de interés para transmitirla de forma inalámbrica, o hacer llegar un delgado cable hasta dicho punto; lo cual revolucionaría sin duda los actuales sistemas de electrodos.

Incluso un micro robot capaz de transmitir información desde un lugar específico a través de diferentes canales (radio, ultrasonido, etc.), podría mejorar la calidad de la información disponible sobre un paciente. En un punto remoto se dispondría del historial de comportamiento de la región de interés (por ejemplo la concentración de oxígeno en una arteria) (Cui et al., 2007), mientras que la ubicación del robot permitiría hallar un fenómeno interno (como un sangrado), informar dicha posición y realizar tareas de medición del fenómeno y su posterior transmisión de alerta (Kim et al., 2007).

En esta tecnología uno de los principales retos existentes es cómo mover al micro robot. Debido a su dimensión, la complejidad de instalar medios de locomoción internos crece exponencialmente a medida que disminuye su tamaño. Es por esto que los sistemas de navegación externos son cada vez más utilizados como solución al problema de navegación. En especial, sistemas electromagnéticos controlados a partir de una corriente de entrada, que modifica los campos magnéticos generados. Uno de ellos, el "OctoMag", es un sistema de cinco grados de libertad que permite manipular un micro robot en su espacio de trabajo a través de campos magnéticos producidos por ocho bobinas (Kummer et al., 2010). Este sistema es utilizado actualmente para estudiar la movilidad y manipulación de un micro robot dentro del humor vítreo de un conejo (Ullrich et al., 2013).

El uso de electroimanes da como ventaja el control directo de los campos magnéticos y gradientes generados, y por ende, un control más preciso del micro robot en el medio. Pero en cambio la desventaja se presenta en la cantidad de energía necesaria para alimentar dichos sistemas, requiriendo equipos dedicados, manejo de altas corrientes y derivado a esto último, alto consumo de potencia y generación de calor, lo cual podría afectar el rendimiento esperado (Dauge et al., 2007; Hagiwara et al., 2011).

La necesidad presente da lugar finalmente al desarrollo de sistemas de arreglos de electroimanes de menor consumo con un control satisfactorio sobre el dispositivo. En este artículo en particular se describe un sistema de navegación magnética basado en el arreglo Helmholtz-Maxwell (Yesin et al., 2006), junto con una técnica de fabricación de micro robots, llevados a cabo en el Instituto de Biorobótica de la Scuola Superiore Sant'Anna, Italia. Igualmente se presenta una herramienta de simulación por software desarrollada para determinar los requerimientos del sistema en diferentes escenarios. Al final se comparan los resultados producto de la experimentación y la simulación para determinar el valor de la herramienta software diseñada.

\section{Materiales y métodos}

Los medios externos de navegación magnética facilitan enormemente el control de posición de los micro robots, con la manipulación directa del campo magnético y el gradiente a través de las corrientes que fluyen por las bobinas. Esto permite modelar matemáticamente el movimiento del micro robot a través de las fuerzas que lo gobiernan y definir su posición a partir de las corrientes de entrada de las bobinas.

\section{Modelado matemático}

Por medio de las ecuaciones de Maxwell es posible definir la navegación magnética de un micro robot a través de la generación de una fuerza $F$ y un torque $T$ que se expresan a continuación (Jiles, 2015):

$$
\begin{gathered}
\vec{F}=\vec{V} \cdot(\vec{M} \cdot \nabla) \vec{B} \\
\vec{T}=V \vec{M} \times \vec{B}
\end{gathered}
$$

Donde $B$ es el campo magnético, $\nabla$ el gradiente, $V$ el volumen del robot y $M$ la magnetización presente en el mismo.

Estas fuerzas son utilizadas en la mayor parte de los sistemas de navegación para micro robots, uti- 
lizando generalmente arreglos de bobinas tipo Helmholtz y Maxwell detallados en la Figura 1 (Choi et al., 2009). Una configuración tipo Helmholtz consiste en dos bobinas circulares idénticas, situadas a lo largo de un eje, separadas por una distancia $d_{h}$ que es igual a su radio $r_{h}$. Aplicando la misma corriente, en la misma dirección, se genera un campo magnético altamente uniforme, que produce un torque sobre el micro robot. En la configuración Maxwell en cambio, la separación de los electroimanes $d_{m}$ es $\sqrt{3} r_{m}$, siendo $r_{m}$ el radio de las bobinas, con el flujo de las corrientes en sentidos opuestos. Esto permite generar un gradiente magnético casi uniforme a lo largo del eje, que se usa para aplicarle una fuerza magnética al robot.

\section{Figura 1. Diagrama de bobinas tipo Helmholtz y Maxwell.} Fuente: elaboración propia.

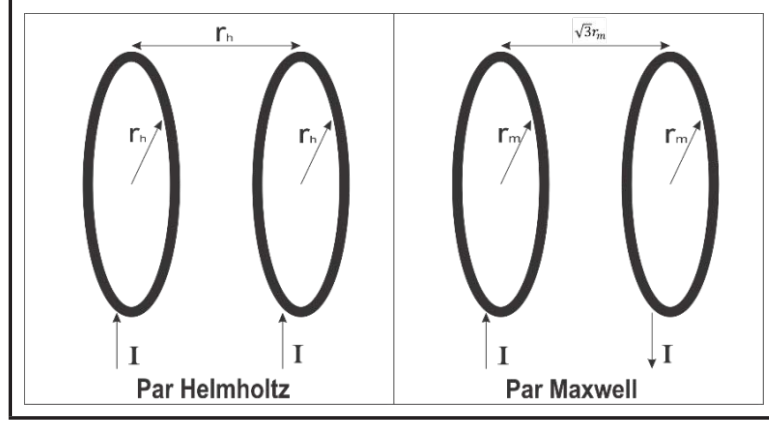

La densidad del flujo magnético y su gradiente, asociados al arreglo de bobinas Helmholtz y Maxwell a lo largo del eje principal, pueden ser aproximados a un valor constante. Por tanto dichas cantidades van a depender directamente de la corriente aplicada al sistema, por las siguientes ecuaciones:

$$
\begin{gathered}
B=\frac{8}{5 \sqrt{5}} \frac{\mu_{o} N_{H}}{R_{H}} \vec{I}=k I_{H} \\
\nabla B=\frac{48 \sqrt{3}}{49 \sqrt{7}} \frac{\mu_{o} N_{M}}{R_{M}{ }^{2}} \vec{I}=g I_{M}
\end{gathered}
$$

Donde $k$ y $g$ son coeficientes proporcionales, definidos por $\mu_{o}$ la permeabilidad magnética en el vacío, $N_{H}$ y $N_{M}$ el número de vueltas y $R_{H}, R_{M}$ el radio de las bobinas para Helmholtz y Maxwell respectivamente.
Con el fin de simplificar el modelo, se asume que el robot es un disco sólido, sumergido en un líquido con un número de Reynolds pequeño (flujo laminar casi estático), perpendicular a su eje. Con esa consideración, la fuerza de arrastre en un espacio cerrado puede ser aproximada por (Kim et al., 2001):

$$
\vec{F}_{D}=\frac{4 \pi \mu_{d} v}{\log \left(\frac{2 d_{0}}{r}\right)-\frac{1}{4}\left(\frac{r}{d_{0}}\right)^{2}} h
$$

Donde $\mu_{d}$ es la viscosidad dinámica del fluido, $v, h$ y $r$ la velocidad, el grosor y el radio del robot respectivamente, y $d_{0}$ la distancia desde el centro del microrobot hasta el espacio donde está confinado. En el plano, las fuerzas presentes sobre el micro robot se resumen a continuación:

$$
\vec{F}_{\text {mag }}+\vec{F}_{D}=m \vec{a}
$$

Donde $\vec{F}_{\text {mag }}$ y $\vec{F}_{D}$ están definidas por (1) y (5) respectivamente, $m$ es la masa del micro robot y $a$ la aceleración en el plano $x y$.

\section{Magnetización}

El desarrollo de materiales compuestos de polímetros embebidos de polvos ferromagnéticos, es una interesante aproximación al desarrollo de micro robots que ofrezcan una buena controlabilidad magnética, al tiempo que se reduce el impacto secundario sobre las células en la región de operación. Es por ello que han sido propuestos varios micro objetos producto de mezclas de polímeros y $\mathrm{NdFeB}$ (iman de neodimio) para la fabricación de sus componentes MEMS (Pawlowski \& Töpfer, 2004).

Para el sistema actual en particular, los micro robots son fabricados a partir del polímero PDMS, por su biocompatibilidad, alta viscosidad (favorable durante la fabricación) y con diferentes concentraciones de polvo de NdFeB. Iacovacci et. al., describen el proceso de fabricación para obtener una delgada película del compuesto, en el rango de 15-35 $\mu \mathrm{m}$ (dependiendo de la concentración del polvo), siendo luego cuidadosamente cortados de forma manual en dimensiones cercanas a los $500 \mu \mathrm{m}$. 
La caracterización para cada concentración de polvo magnético en el polímero se realiza de manera experimental, aplicando un campo de excitación $H$, para obtener la magnetización $M$ del compuesto. La variación en la concentración de $\mathrm{NdFeB}$, describe diferentes curvas en el comportamiento magnético, la susceptibilidad y saturación magnética (Iacovacci et al., 2015).

\section{Experimentación y desarrollo}

El proceso de experimentación se llevó a cabo en el laboratorio del Instituto de Biorobótica de la Scuola Superiore Sant'Anna, ubicado en Pontedera, Pisa, Italia. Durante la estancia llevada a cabo por el autor principal de este artículo, se tuvo acceso al sistema de electroimanes presente (Figura 6), recogiendo suficiente información para comparar el modelo de simulación, complementando con la elaboración de nuevos micro robots realizando el procedimiento mencionado anteriormente.

Unity3D es un motor gráfico ampliamente utilizado para videojuegos. Incluye un motor de físicas decente y es muy versátil al incluir nuevas funciones de trabajo. A pesar de no ser una herramienta ideal para realizar simulaciones físicas, resulta bastante interesante evaluar las capacidades de modelamiento matemático a la vez que ofrece un resultado gráfico bastante llamativo. Dentro de los objetivos de este trabajo, se deseaba evaluar igualmente el potencial de la herramienta, con el fin de aprovechar la facilidad de manipulación de objetos 3D, que aportarán sin duda más datos a investigaciones de este tipo.

Dentro del plan propuesto, la comparación se realizaría con la respuesta del sistema a través de la velocidad obtenida por las distintas concentraciones de polvo magnético presente en el micro robot. Esto permitiría definir si la herramienta tiene el potencial esperado para dichos experimentos y posteriormente, ser capaz de simular escenarios más complejos, haciendo símil a un ambiente real de aplicación médica. El enfoque en estos nuevos escenarios supone determinar nuevas características físicas y técnicas necesarias para utilizar un sistema de navegación electromagnético en el cuerpo humano, con el fin de encontrar los requerimientos y limitaciones de energía para la navegación del robot.

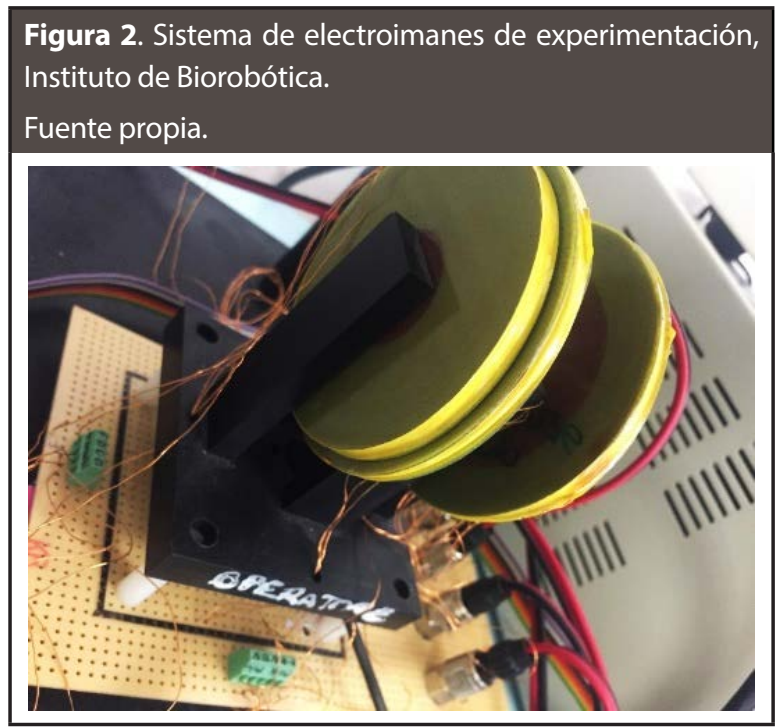

\section{Modelado del sistema para} simulación

Para la simulación del sistema se utilizó el arreglo de bobinas Helmholtz y Maxwell presente en el laboratorio (Figura 2), con las características resumidas en la Tabla 1. En ella se encuentran las dimensiones para las bobinas tipo Helmholtz y tipo Maxwell en los ejes X e Y, número de vueltas y el cálculo de las constantes $k$ y $g$ de acuerdo a las Ecuaciones (3) y (4).

TABLA 1. CARACTERÍSTICAS DEL SISTEMA MAGNÉTICO.

\begin{tabular}{c|c|c|c|c} 
& Hx & Hy & Mx & My \\
\hline Radio interno (mm) & 25,16 & 7,54 & 12,46 & 8,16 \\
\hline Radio externo (mm) & 36,04 & 11,06 & 32,94 & 12.64 \\
\hline Número de vueltas & 340 & 110 & 640 & 140 \\
\hline $\mathbf{k}(\mathrm{mT} / \mathrm{A})$ & 6,18 & 6,7 & & \\
\hline $\mathbf{g}(\mathrm{T} /(\mathrm{mA}))$ & & & 0,65 & 0,69 \\
\hline
\end{tabular}

Adicionalmente para llevar a cabo el experimento está presente un $\mathrm{PC}$, una cámara de video (BASLER scA1390-17gc), una tarjeta de adquisición 
de datos (NI DAQ USB-6259), el sistema de alimentación de las bobinas, y un micro ambiente para contener el líquido (aproximadamente $7 \mu \mathrm{L}$ ), léase arena. El control, la interfaz y recopilación de datos se realiza con LabVIEW(C.

\section{Resultados}

El proceso de experimentación consistió en introducir el micro robot en la arena y efectuar pruebas de movimiento a través de la inyección de la corriente necesaria para obtener un campo magnético y gradiente máximo de $8 \mathrm{mT}$ y $0.6 \mathrm{~T} / \mathrm{A}$ respectivamente, generando el torque y la fuerza magnética necesaria para el desplazamiento controlado del micro robot. La cámara realiza registros (fotos) 100 veces por segundo, similares al mostrado en la Figura 3, que son almacenados en el PC. Dichos registros son posteriormente analizados con el fin de determinar las velocidades alcanzadas por el micro robot, a través de un algoritmo de imagen utilizando MatLab®. Esta variable fue utilizada para comparar la respuesta obtenida en el modelo de simulación.

El modelado 3D de las características físicas se realizó en el software CAD SolidWorks®, posteriormente fue exportado al motor gráfico Unity3D. La escena contiene las medidas exactas del modelo real con el fin de tener una mayor correspondencia en la física completa del sistema (Figura 2).

Figura 3. Registro de la cámara: Visualización de la arena y el micro robot.

Fuente propia.

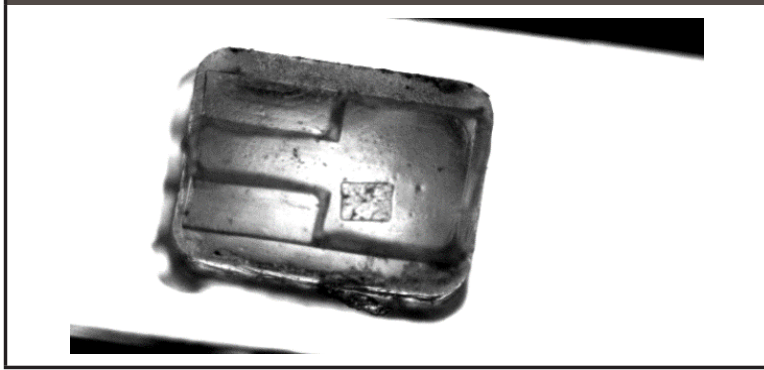

El comportamiento magnético del micro robot se modela a partir de dos scripts en C\#, para el torque y la fuerza magnética presente, que corren simultáneamente. En la escena se presentan indicadores de las variables utilizadas en el sistema, leídas directamente de dichos scripts. Por último la variable de salida (velocidad) se almacena en un documento de texto para su análisis posterior. La escena queda dividida en tres cámaras (Figura 4), a la izquierda el comportamiento del micro robot, a la derecha arriba la vista general del sistema y a la derecha abajo la referencia u orientación. El control del sistema se realiza con un joypad, aunque también es posible utilizar el teclado.

Figura 4. Escena de simulación en Unity3D.

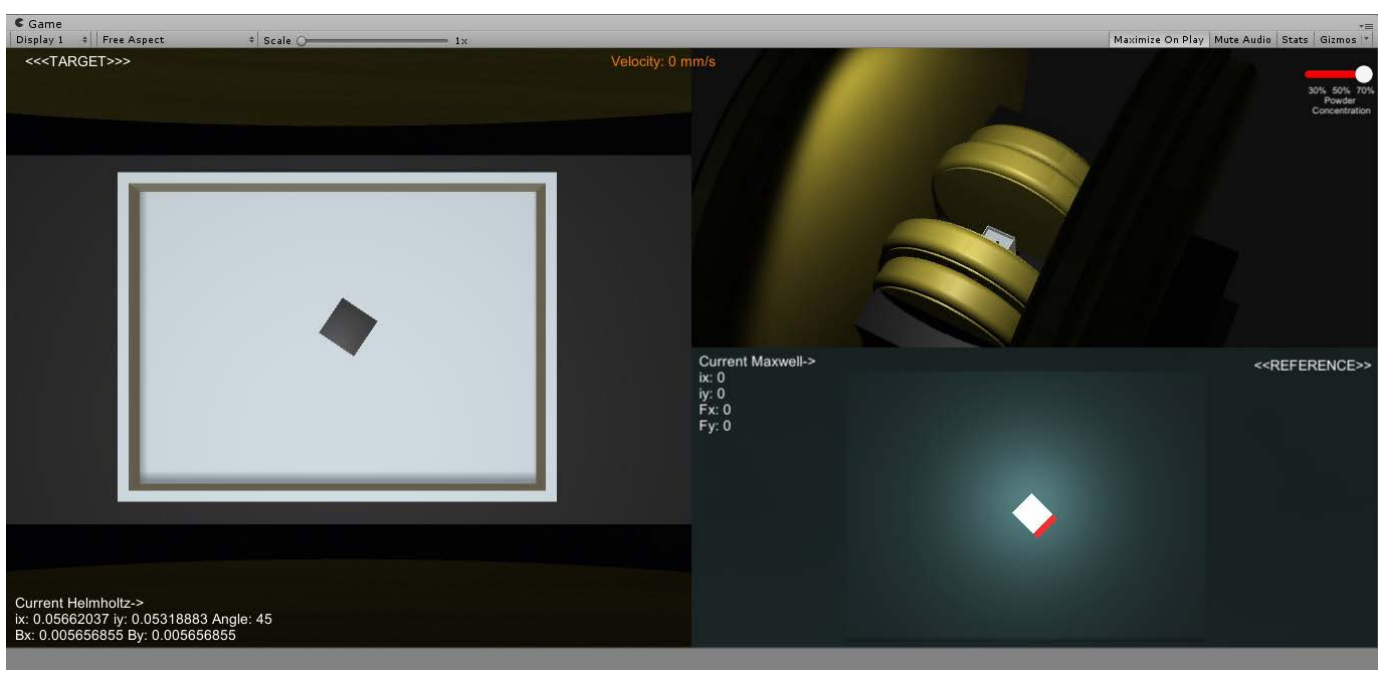


Efectuando repetidas veces el proceso de simulación, los datos se almacenan con el fin de compararlos con la respuesta de la experimentación. Para ello se utilizan tres concentraciones de polvo magnético (30\%, 50\% y 70\%), obteniéndose sus respectivas curvas de magnetización (Figura 5), con el fin de variar los parámetros de entrada en la simulación, donde se observa su comportamiento y su fiabilidad al modelo real. La variable de salida analizada es la velocidad, que está directamente ligada a la concentración magnética de cada elemento.

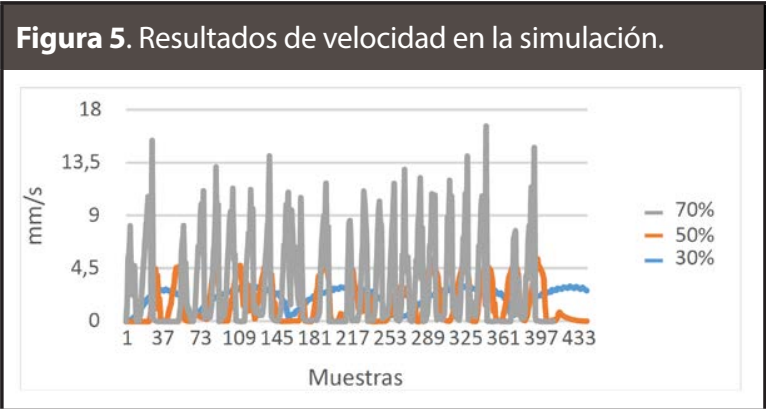

Figura 6. Resultados de simulación vs experimentación.

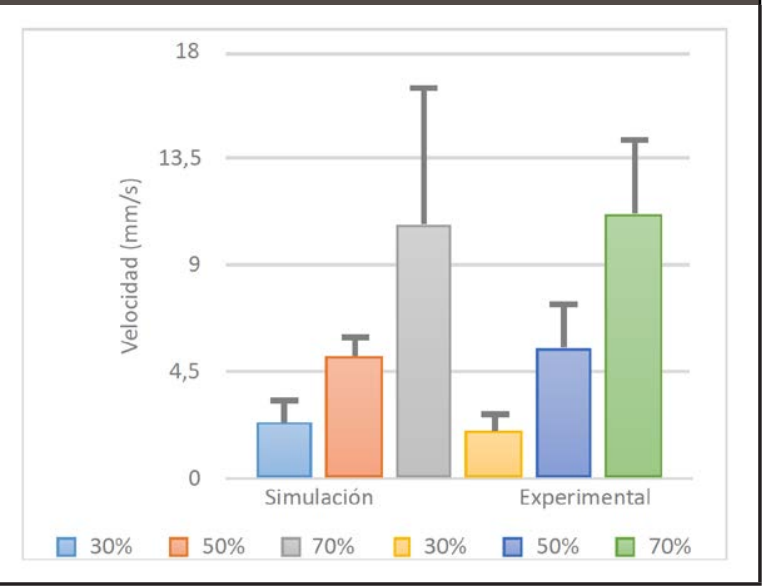

En la Figura 6 se comparan los resultados promedio obtenidos en la simulación (izquierda), con los valores promedio registrados en la experimentación (derecha), para cada concentración de NdFeB. Los resultados en la simulación varían conforme la cantidad de concentración presente en el material, mostrando una clara correlación entre la velocidad y la concentración magnética, tal como en el sistema real. De igual manera, una mayor concentración ob- tendrá mejores respuestas en desplazamiento, pero una mayor dificultad de control de posición.

En la siguiente tabla se resumen los valores promedio de velocidad, obtenidos tanto en experimentación como en simulación. La desviación media obtenida en los resultados es de alrededor de 0.2 $\mathrm{mm} / \mathrm{s}$, un valor bastante bueno si se compara con modelos de simulación actuales en estos escenarios (Arcese et al., 2013).

TABLA 2. COMPARATIVA DE DATOS Y DESVIACIONES.

\begin{tabular}{|c|c|c|c|}
$\begin{array}{c}\text { Concentración } \\
\text { de NdFeB }\end{array}$ & $\begin{array}{c}\text { Velocidad } \\
\text { simulación } \\
\text { promedio } \\
(\mathrm{mm} / \mathrm{s})\end{array}$ & $\begin{array}{c}\text { Velocidad } \\
\text { experimentación } \\
\text { promedio }(\mathrm{mm} / \mathrm{s})\end{array}$ & Desviación \\
\hline $30 \%$ & 2,20 & 1,99 & 0,21 \\
\hline $50 \%$ & 5,26 & 5,55 & $-0,29$ \\
\hline $70 \%$ & 11,93 & 11,22 & 0,71 \\
\hline & & $\begin{array}{c}\text { DESVIACIÓN } \\
\text { MEDIA }\end{array}$ & 0,21 \\
\hline
\end{tabular}

\section{Conclusiones}

Los resultados obtenidos dan cuenta del potencial de la herramienta de simulación para la planeación de sistemas en escenarios diversos. Esto significa que a través de este modelo, es posible prever recursos y requisitos de funcionamiento en la navegación electromagnética de micro robots en un ambiente determinado. De igual forma, podría establecerse la base para el desarrollo de un sistema de planeación operativa de micro robots en un ambiente quirúrgico futuro, tales como el punto de inserción, el recorrido más favorable, etc.

Aunque el comportamiento físico está principalmente modelado matemáticamente en el código de la simulación, es posible que se presenten algunas limitaciones en casos especiales debido a las aproximaciones del modelo, restricciones de programación de máquina o del motor físico incluido en Unity3D. Aún con esto el rendimiento del sistema modelado mantiene una clara correlación a la variación de concentración magnética del micro robot 
observada en el proceso de experimentación, que a su vez coincide con los resultados de Iacovacci (Iacovacci et al., 2015).

Aunque hay un campo bastante interesante en el uso de Unity3D como plataforma de simulación para nuevos sistemas electromagnéticos, cabe destacar la necesidad emergente de sistemas de planeación para la navegación de micro robots en un entorno operativo de medicina. La versatilidad del motor gráfico y las propiedades del motor físico, facilitarían en gran medida el desarrollo de dichas aplicaciones, a la par que ofrecerían una visualización en tiempo real del comportamiento del micro robot. Igualmente es un campo bastante amplio en el que las herramientas de simulación tendrán un gran aporte en la operatividad de los micro robots para usos médicos. Todo esto hace parte de los futuros trabajos que serán abordados con esta herramienta.

\section{Agradecimientos}

Los autores agradecen a los doctores Gioia Lucarini y Veronica Iaccovacci, así como al Instituto de Biorobótica de la Scuola Superiore de Sant'Anna, en Italia, por el apoyo brindado en su laboratorio con el fin de desarrollar esta investigación.

\section{Referencias}

Andrä, W., \& Nowak, H. (2007). Magnetism in Medicine: A Handbook. Weinheim: Wiley.

Arcese, L., Fruchard, M., \& Ferreira, A. (2013). Adaptive Controller and Observer for a Magnetic Microrobot. IEEE Transactions on Robotics, 29(4), 1060-1067.

Chatzipirpiridis, G., Ergeneman, O., Pokki, J., Ullrich, F., Fusco, S., Ortega, J. A., Pane, S. (2015). Electroforming of implantable tubular magnetic microrobots for wireless ophthalmologic applications. Advanced Healthcare Materials, 4(2), 209-214. https://doi. org/10.1002/adhm.201400256

Choi, H., Choi, J., G, J., Park, J. O., \& Park, S. (2009). Twodimensional actuation of a microrobot with a stationary two-pair coilsystem. Smart Materials and Structures, 18(5), 1-9.

Cui, J., Wei, Y., \& Wang, H. (2007). The study of a telemetry robot for gastrointestinal tract. In M. Sasaki, G. Choi
Sang, Z. Li, R. Ikeura, H. Kim, \& F. Xue (Eds.) (Vol. 6794, p. 67943G). https://doi.org/10.1117/12.783873

Dario, P., Carrozza, M. C., Benvenuto, A., \& Menciassi, A. (2000). Micro-systems in biomedical applications. Journal of Micromechanics and Microengineering, 10(2), 235-244. https://doi.org/10.1088/0960$1317 / 10 / 2 / 322$

Dauge, M., Gauthier, M., \& Piat, E. (2007). Modelling of a planar magnetic micropusher for biological cell manipulations. Sensors and Actuators A: Physical, 138(1), 239-247. https://doi.org/10.1016/j. sna.2007.04.057

Ergeneman, O., Abbott, J. J., Dogangil, G., \& Nelson, B. J. (2008). Functionalizing intraocular microrobots with surface coatings. In 2008 2nd IEEE RAS \& EMBS International Conference on Biomedical Robotics and Biomechatronics (pp. 232-237). https://doi. org/10.1109/BIOROB.2008.4762857

Hagiwara, M., Kawahara, T., Yamanishi, Y., \& Arai, F. (2011). Precise Control of Magnetically Driven Microtools for Enucleation of Oocytes in a Microfluidic Chip. Advanced Robotics, 25(8), 991-1005. https://doi. org/10.1163/016918611X568611

Iacovacci, V., Lucarini, G., Innocenti, C., Comisso, N., Dario, P., Ricotti, L., \& Menciassi, A. (2015). Polydimethylsiloxane films doped with $\mathrm{NdFeB}$ powder: magnetic characterization and potential applications in biomedical engineering and microrobotics. Biomedical Microdevices, 17, 112.

Jiles, D. (2015). Introduction to Magnetism and Magnetic Materials, Third Edition. London: CRC Press.

Kim, D.-H., Haake, A., Sun, Y., Neild, A., Ihm, J., Dual, J., ... Nelson, B. (2004). High-throughput cell manipulation using ultrasound fields. Annual International Conference of the IEEE Engineering in Medicine and Biology - Proceedings.

Kim, M. U., Kim, K. W., Cho, Y. H., \& Kwak, B. M. (2001). Hydrodynamic force on a plate near the plane wall. Part I: plate in sliding motion. Fluid Dynamics Research, 29(3), 137.

Kim, T. S., Song, S. Y., Jung, H., Kim, J., \& Yoon, E. S. (2007). Micro Capsule Endoscope for Gastro Intestinal Tract. In 2007 29th Annual International Conference of the IEEE Engineering in Medicine and Biology Society (pp. 2823-2826). https://doi.org/10.1109/ IEMBS.2007.4352916 
Kummer, M. P., Abbott, J. J., Kratochvil, B. E., Borer, R., Sengul, A., \& Nelson, B. J. (2010). OctoMag: An Electromagnetic System for 5-DOF Wireless Micromanipulation. IEEE Transactions on Robotics, 26(6), 1006-1017. https://doi.org/10.1109/ TRO.2010.2073030

Lai, J.-Y., Tsai, N.-C., \& Chiu, H.-L. (2014). Theoretical analysis and simulations of micro-dosing locomotive robot with drug-release mechanism. Robotics and $\mathrm{Au}$ tonomous Systems, 62(2), 177-187. https://doi.org/ https://doi.org/10.1016/j.robot.2013.11.001

Luechinger, N. A., Walt, S. G., \& Stark, W. J. (2010). Printable Nanoporous Silver Membranes. Chemistry of Materials, 22(17), 4980-4986. https://doi.org/10.1021/ cm1013152

Mack, M. J. (2001). Minimally invasive and robotic surgery. JAMA, 285(5), 568-572.

Menciassi, A., Quirini, M., \& Dario, P. (2007). Microrobotics for future gastrointestinal endoscopy. Minimally Invasive Therapy \& Allied Technologies: MITAT: Official Journal of the Society for Minimally Invasive Therapy, 16(2), 91-100. https://doi. org/10.1080/13645700701266982

Park, S., Cha, K., \& Park, J. (2010). Development of Biomedical Microrobot for Intravascular Therapy. International Journal of Advanced Robotic Systems, 7(1), 1. https://doi.org/10.5772/7260

Pawlowski, B., \& Töpfer, J. (2004). Permanent magnetic NdFeB thick films. Journal of Materials Science, 39(4), 1321-1324. https://doi.org/10.1023/ B:JMSC.0000013891.38980.1c

Phillip, M., \& Devlin, M. D. F. (2015). Brachytherapy, Second Edition: Applications and Techniques. Springer Publishing Company. Retrieved from https://books. google.com.co/books?id=MFfSCgAAQBAJ

Qiu, F., Zhang, L., Tottori, S., Marquardt, K., Krawczyk, K., Franco-Obregón, A., \& Nelson, B. J. (2012). Bio-inspired microrobots. Materials Today, 15(10), 463. https://doi.org/https://doi.org/10.1016/S13697021(12)70201-8

Saito, S., Tanaka, S., Hiroe, Y., Miyashita, Y., Takahashi, S., Satake, S., \& Tanaka, K. (2003). Angioplasty for chronic total occlusion by using tapered-tip guidewires. Catheterization and Cardiovascular Interventions: Official Journal of the Society for Cardiac Angiography \& Interventions, 59(3), 305-311. https:// doi.org/10.1002/ccd.10505
Shah, B. C., Buettner, S. L., Lehman, A. C., Farritor, S. M., \& Oleynikov, D. (2009). Miniature in vivo robotics and novel robotic surgical platforms. The Urologic Clinics of North America, 36(2), 251-63, x. https://doi. org/10.1016/j.ucl.2009.02.013

Ullrich, F., Bergeles, C., Pokki, J., Ergeneman, O., Erni, S., Chatzipirpiridis, G., ... Nelson, B. J. (2013). Mobility experiments with microrobots for minimally invasive intraocular surgery. Investigative Ophthalmology \& Visual Science, 54(4), 2853-2863. https://doi. org/10.1167/iovs.13-11825

V Joseph, J., Arya, M., \& R H Patel, H. (2005). Robotic surgery: The coming of a new era in surgical innovation. Expert Review of Anticancer Therapy, 5, 7-9.

Watson, B., Friend, J., Yeo, L., \& Sitti, M. (2009). Piezoelectric ultrasonic resonant micromotor with a volume of less than $1 \mathrm{~mm} 3$ for use in medical microbots. Proceedings - IEEE International Conference on Robotics and Automation.

Woods, S. P., \& Constandinou, T. G. (2013). Wireless capsule endoscope for targeted drug delivery: mechanics and design considerations. IEEE Transactions on Bio-Medical Engineering, 60(4), 945-953. https:// doi.org/10.1109/TBME.2012.2228647

Yesin, K. B., Vollmers, K., \& Nelson, B. J. (2006). Modeling and Control of Untethered Biomicrorobots in a Fluidic Environment Using Electromagnetic Fields. The International Journal of Robotics Research, 25(5-6), 527-536. https://doi. org/10.1177/0278364906065389

Zhang, H., Hutmacher, D. W., Chollet, F., Poo, A. N., \& Burdet, E. (2005). Microrobotics and MEMS-based fabrication techniques for scaffold-based tissue engineering. Macromolecular Bioscience, 5(6), 477-489. https://doi.org/10.1002/mabi.200400202

\section{PARA CITAR ESTE ARTÍCULO / TO REFERENCE THIS ARTICLE / PARA CITAR ESTE ARTIGO /}

Muñoz Medina, M.A.; Vivas, O.A.; Riccotti, L. (2018). Herramienta para la simulación del movimiento de un micro robot para aplicaciones médicas a partir de un arreglo de bobinas basadas en Maxwell - Helmholtz. (Herramienta de simulación para navegación de microrobots). Revista EIA, 15(30), julio-diciembre, pp. 151-160. [Online]. Disponible en: https://doi.org/10.24050/reia.v15i30.1254 